\title{
Trifluridine/tipiracil increases survival rates in peritoneal dissemination mouse models of human colorectal and gastric cancer
}

\author{
NORIHIKO SUZUKI $^{1}$, FUMIO NAKAGAWA ${ }^{2}$ and TEIJI TAKECHI ${ }^{1}$ \\ ${ }^{1}$ Translational Research Laboratory; ${ }^{2}$ Applied Pharmacology Laboratory, \\ Tokushima Research Center, Taiho Pharmaceutical Co., Ltd., Tokushima 771-0194, Japan
}

Received August 25, 2016; Accepted March 7, 2017

DOI: $10.3892 / \mathrm{ol} .2017 .6258$

\begin{abstract}
A number of patients exhibit peritoneal dissemination of gastric or colorectal cancer, which is a predominant cause of cancer-associated mortality. Currently, there is no markedly effective treatment available. The present study was designed to determine the efficacy of trifluridine/tipiracil (TFTD), formerly known as TAS-102, which is used for the treatment of patients with unresectable advanced or recurrent colorectal cancer refractory to standard therapies. Four colorectal cancer cell lines and one gastric cancer cell line were intraperitoneally inoculated into nude mice, as models of peritoneal dissemination. TFTD (200 mg/kg/day) was orally administered for 5 consecutive days followed by 2 drug-free days for 6 weeks. The increase in the lifespan (ILS) of the TFTD-treated mice compared with that of the drug-free control mice was $66.7,43.3,106.3,98.3$ and $133.3 \%$ for DLD-1, DLD-1/5-fluorouracil [5-fluorouracil (5FU)-resistant subline of DLD-1], HT-29 and HCT116 colorectal cancer cell lines, and MKN45 gastric cancer cell line, respectively. This ILS was similar to that of the irinotecan-treated mice (ILS, $70-84 \%)$, but was significantly $(\mathrm{P}<0.05)$ increased compared with that of the 5FU-, tegafur, gimeracil and potassium oxonate- and cisplatin-treated mice (ILS, 1-53\%, 0.8-60\% and $85 \%$, respectively). No significant increase in body weight loss was observed during the dosing periods with any of the drugs used. The increase in CEA levels with progressive peritoneal dissemination was inhibited by TFTD treatment. TFTD also
\end{abstract}

Correspondence to: Dr Norihiko Suzuki, Translational Research Laboratory, Taiho Pharmaceutical Co., Ltd., 224-2 Ebisuno Hiraishi, Kawauchi-Cho, Tokushima, Tokushima 771-0194, Japan

E-mail: n-suzuki@taiho.co.jp

Abbreviations: 5FU, 5-fluorouracil; BSC, best supportive care; CEA, carcinoembryonic antigen; CPT-11, irinotecan; ILS, increase in lifespan; OS, overall survival; TFTD, trifluridine and tipiracil; TPI, tipiracil

Key words: colorectal cancer, gastric cancer, increase in lifespan, TAS-102, trifluridine, tipiracil exhibited marked anticancer effects against Kirsten rat sarcoma viral oncogene homolog-mutated tumors and 5FU-resistant tumors. The results of the present study indicate that TFTD may be a potential drug against peritoneal dissemination of colorectal and/or gastric cancer in humans and may be utilized for chemo-naïve tumors and recurrent tumors following 5FU treatment.

\section{Introduction}

The most critical and ultimately life-threatening characteristic of malignant cancers is the ability to metastasize to other organs (1). Between 4 and $7 \%$ of patients with colon cancer present with peritoneal carcinomatosis at the time of diagnosis, despite advances in early detection of the disease (2). Systemic chemotherapeutic agents, including 5-fluorouracil (5FU), 5FU derivatives, irinotecan (CPT-11) and oxaliplatin, as well as targeted agents including bevacizumab, cetuximab, panitumumab and regorafenib are currently used for the treatment of unresectable metastatic colorectal cancer, and the survival time of patients with unresectable metastatic colorectal cancer has been improved $(3,4)$. However, as almost all patients involved in these studies are patients with liver or lung metastases, the effects of these agents on peritoneal dissemination remain unclear $(5,6)$. In Phase III trials $(7,8)$, systemic chemotherapy, including the 5FU-based combination therapy folinic acid-5-FU-oxaliplatin, was not identified to be effective against peritoneal dissemination and failed to significantly improve survival (9). Peritoneal metastasis of gastric cancer also leads to poor clinical outcomes, particularly with serosal exposure and undifferentiated histology (10). For unresectable or recurrent gastric cancer, only a limited number of Phase II studies have examined the efficacy of these regimens for peritoneal metastasis $(11,12)$. In spite of recent advances in systemic chemotherapy, peritoneal dissemination due to colorectal or gastric cancer remains a dismal disease with a markedly low survival rate in patients.

Trifluridine/tipiracil (TFTD), formerly known as TAS-102, is an antitumor therapy $(13,14)$. It comprises a mixture of two distinct chemicals, trifluridine and tipiracil (TPI), at a molar ratio of 1:0.5. Trifluridine, an analog of thymidine, exerts its antitumor activity through two mechanisms. It inhibits 
thymidylate synthase (15) and is incorporated into DNA (16). TPI enhances the bioavailability of trifluridine by inhibiting its in vivo degradation by thymidine phosphorylase. TPI is therefore beneficial by producing a more durable and sustainable response to trifluridine (17).

The antitumor effects of TFTD on colon cancer xenograft in models refractory to $5 \mathrm{FU}$ are hypothesized to primarily involve trifluridine incorporation into DNA (18). At the twice-daily oral dosing, the clinically used administration schedule, the primary TFTD cytotoxic mechanism is also hypothesized to be mediated by DNA incorporation of trifluridine (19). TFTD significantly improved the overall survival (OS) period [median OS, 7.1 months; 95\% confidence interval (CI), 6.5-7.8 months vs. median OS, 5.3 months; 95\% CI, 4.6-6 months for placebo) and progression-free survival (PFS) in patients with metastatic colorectal cancer refractory to standard chemotherapies as demonstrated by an international multi-center randomized double-blind Phase III study (20). The same study also demonstrated that TFTD exhibits a favorable safety profile. These results led to the regulatory approval of the drug in the USA and, recently, in Europe. With regard to gastric cancer, a multicenter Phase II study demonstrated that TFTD exhibited a positive efficacy and an acceptable toxicity profile in patients with advanced gastric cancer following progression on one or two prior systemic chemotherapies (21). A randomized double-blind Phase III study evaluating TFTD plus best supportive care (BSC) compared with placebo plus $\mathrm{BSC}$ in patients with metastatic gastric cancer refractory to standard treatments is currently ongoing (22).

In the present study, the effects of TFTD on the survival times of mice inoculated with colorectal or gastric cancer cells into the peritoneal cavity were evaluated in comparison with other drugs. The present study may provide useful information to improve and/or expand options for the treatment of human colorectal and gastric cancers.

\section{Materials and methods}

Reagents. TFTD, and tegafur, gimeracil and potassium oxonate (S-1) were obtained from Taiho Pharmaceutical Co., Ltd. (Tokyo, Japan). CPT-11 was purchased from Yakult Honsha Co., Ltd. (Tokyo, Japan). 5FU was purchased from Kyowa Hakko Kirin Co., Ltd. (Tokyo, Japan). Cisplatin (CDDP) was purchased from Nippon Kayaku Co., Ltd. (Tokyo, Japan). Hydroxypropyl methylcellulose (HPMC) was obtained from Shin-Etsu Chemical Co., Ltd. (Tokyo, Japan).

Cancer cell lines. The human colon cancer cell line HT-29 was purchased from the American Type Culture Collection (Manassas, VA, USA). Human colorectal carcinoma DLD-1 cells and HCT116 cells were purchased from Dainippon Sumimoto Pharma Co., Ltd. (Osaka, Japan). The 5FU-resistant cell line DLD-1/5FU was established using long-term culture in the presence of $5 \mathrm{FU}$ in vitro (23). The human gastric cancer cell line MKN45 was purchased from RIKEN BioResource Center Cell Bank (Tsukuba, Japan) (24).

The cell lines were cultured in RPMI-1640 medium (HT-29, DLD-1, DLD-1/5FU and MKN45) or Dulbecco's modified Eagle's medium (HCT116) supplemented with $10 \%$ fetal bovine serum (FBS) at $37^{\circ} \mathrm{C}$ in a humidified atmosphere containing $5 \% \mathrm{CO}_{2}$. Culture media and FBS were obtained from Sigma-Aldrich; Merck KGaA (Darmstadt, Germany). The Kirsten rat sarcoma viral oncogene homolog (KRAS) mutation status of HT-29 and MKN45 was wild-type, whereas DLD-1 and HCT116 are KRAS mutants. The v-Raf murine sarcoma viral oncogene homolog B (BRAF) mutation status of DLD-1, HCT116 and MKN45 was wild-type, whereas HT-29 is a BRAF mutant. The p53 mutation status of DLD-1, HT-29 and MKN45 was wild-type, whereas HCT116 is a p53 mutant.

Animals. Five-week-old male nude mice (BALB/c $n u / n u$; body weight range, 26-31 g, 50 mice in each experiment) were purchased from CLEA Japan, Inc. (Tokyo, Japan) and housed under specific pathogen-free conditions. Food and water were provided ad libitum. Testing rooms were maintained on a $12 \mathrm{~h} \mathrm{light/dark} \mathrm{cycle} \mathrm{(lights} \mathrm{on} \mathrm{at} \mathrm{06:00} \mathrm{a.m.,} \mathrm{lights} \mathrm{out} \mathrm{at}$ 06:00 p.m.) at a temperature of $23 \pm 3^{\circ} \mathrm{C}$. All animal studies were performed according to the guidelines and with the approval of the Institutional Animal Care and Use Committee of Taiho Pharmaceutical Co., Ltd.

In vivo antitumor activity. Four colorectal cancer cell and one gastric cancer cell suspensions were prepared from in vitro cell culture. Each group consisted of 10 animals, into the peritoneal cavity of which were injected $2 \times 10^{7}$ cells for the DLD-1, DLD-1/5FU and HT-29 colorectal cancer cell lines, and MKN45 gastric cancer cell line, or $5 \times 10^{6}$ cells for the HCT116 colorectal cancer cell line. The drug treatment began 3 or 4 days later and was considered as day 1 . For the control group, vehicle $(0.5 \%$ HPMC solution, $10 \mathrm{ml} / \mathrm{kg})$ was orally administered once daily for 5 consecutive days followed by 2 drug-free days for 6 weeks.

TFTD (200 mg/kg/day) was orally administered twice daily for 5 consecutive days followed by 2 drug-free days for 6 weeks (18) and S-1 (10 mg/kg/day) was orally administered once daily for 5 consecutive days followed by 2 drug-free days for 6 weeks (25). 5FU (12 mg/kg) was injected three times a week every 3 weeks for the 6-week period (days 3, 4, 5, 25, 26 and 27) (26). CPT-11 (100 mg/kg) was injected intravenously once-weekly into the tail vein of mice during the 6 -week period for evaluating the effect on the colorectal cancer cell lines (27). CDDP ( $7 \mathrm{mg} / \mathrm{kg}$ ) was injected intravenously once every 3 weeks into the tail vein of mice (days 3 and 31) for evaluating the effect on the gastric cancer MKN45 cell line (28).

The median increase in lifespan (ILS) was calculated as a survival index according to the following formula: ILS $(\%)=[($ median survival time of treated group $) /($ median survival time of control group)-1]x100.

Measurement of carcinoembryonic antigen (CEA) levels in peritoneal dissemination. On days 10, 20, 30, 40 and 50, the mice were sacrificed by cervical dislocation, and $0.5 \mathrm{ml}$ saline was injected into the peritoneal cavity. After $5 \mathrm{~min}, 0.1 \mathrm{ml}$ peritoneal fluid was recovered by abdominal puncture with a $21 \mathrm{G}$ needle. Following centrifugation at 2,300 x $\mathrm{g}$ for $5 \mathrm{~min}$, CEA levels were measured in the supernatant of each sample using a CEA ELISA kit (DRG Instruments GmbH, Marburg, Germany).

Statistical analysis. The difference in the survival period distribution among groups was analyzed using the log-rank 
A

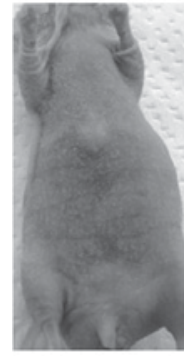

Normal

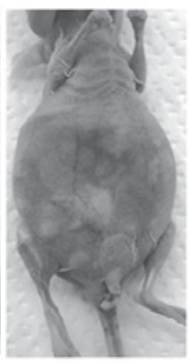

DLD-1

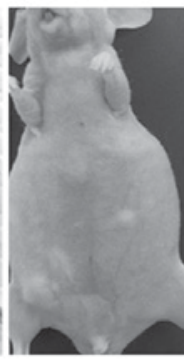

HT-29

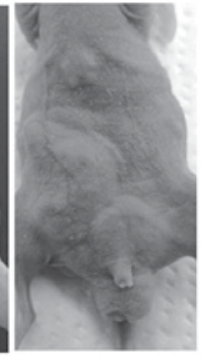

HCT116

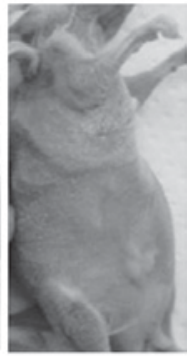

MKN45

B

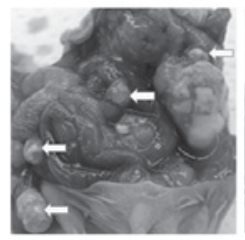

DLD-1

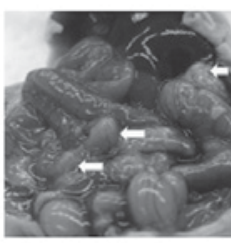

HT-29

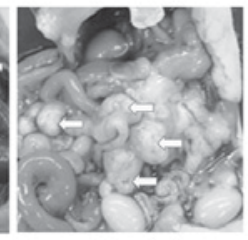

HCT116

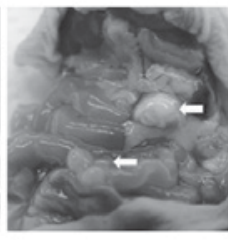

MKN45

Figure 1. Evaluation of human colorectal and gastric tumor intraperitoneal xenograft models. DLD-1, HT-29 and MKN45 cells (2x10 ${ }^{7}$ cells/mouse), and HCT116 cells $\left(5 \times 10^{6}\right.$ cells/mouse) were injected intraperitoneally to generate peritoneal dissemination. (A) General appearance of the mice following intraperitoneal inoculation of DLD-1, HT-29, HCT116 and MKN45 cells at 50, 40, 30 and 30 days, respectively. Carcinomatous peritonitis with an increased amount of bloody ascites for DLD-1 and HT-29 cells or with a limited amount of bloody ascites for HCT116 and MKN45 cells was observed. (B) Intra-abdominal appearance of the mice following intraperitoneal inoculation of DLD-1, HT-29, HCT116 and MKN45 cells at 50, 40, 30 and 30 days, respectively. Arrowheads indicate disseminated tumors throughout the murine peritoneal cavity.
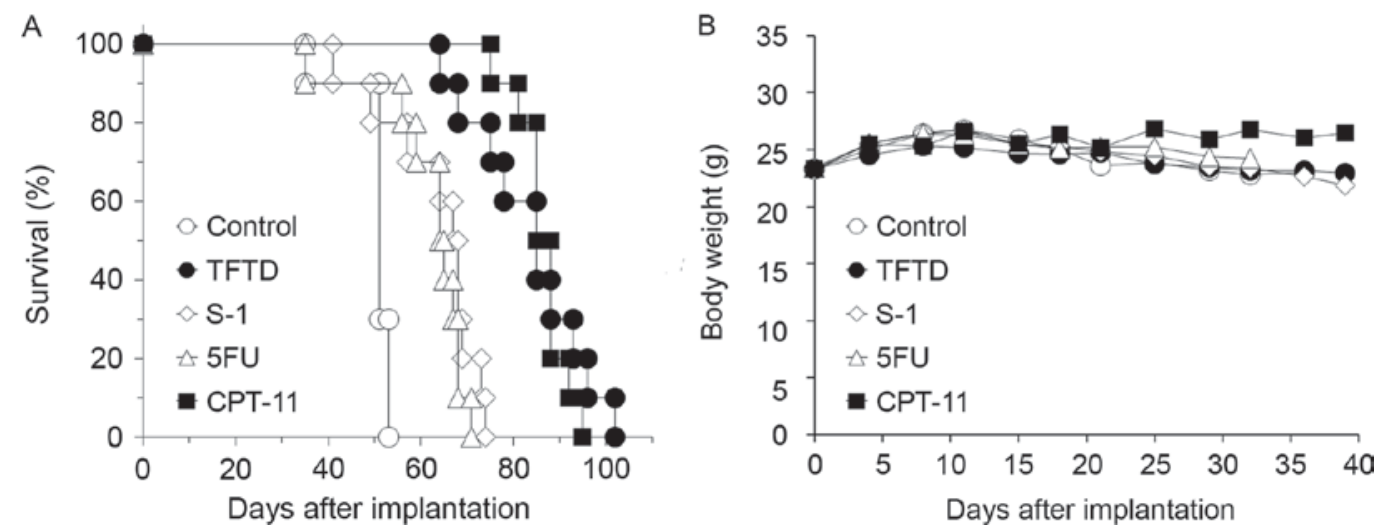

Figure 2. Effect of TFTD (A) on the survival intraperitoneally transplanted with human colorectal cancer DLD-1 cells and on the (B) body weight of DLD-1 tumor-bearing nude mice. Mice were treated with (०) vehicle, $(\bullet)$ TFTD $(200 \mathrm{mg} / \mathrm{kg}$, orally twice daily for 5 consecutive days followed by 2 drug-free days for 6 weeks), $(\diamond) \mathrm{S}-1$ (10 mg/kg/day, orally once daily for 5 consecutive days followed by 2 drug-free days for $6 \mathrm{weeks}),(\Delta) 5 \mathrm{FU}(12 \mathrm{mg} / \mathrm{kg}$, intravenous injection three times a week every 3 weeks for 6 weeks) and (a) CPT-11 (100 mg/kg, intravenously once-weekly during 6 weeks). Body weight was measured twice weekly. Results are presented as means $(\mathrm{n}=10)$. TFTD, trifluridine/tipiracil; S-1, tegafur, gimeracil and potassium oxonate; 5FU, 5-fluorouracil; CPT-11, irinotecan.

test. All statistical analyses were performed using EXSUS (version 8.1; CAC Exicare Corp., Osaka, Japan). $\mathrm{P}<0.01$ was considered to indicate a statistically significant difference.

\section{Results}

Establishment of human colorectal and gastric tumor intraperitoneal xenograft models. Mice inoculated with DLD-1, HT-29, HCT116 and MKN45 cell suspensions were sacrificed for macroscopic examination to determine the distribution of the intraperitoneal dissemination at 50, 40, 30 and 30 days, respectively. Bloody ascites were observed in the mice from the control group following intraperitoneal inoculation with DLD-1 and HT-29 cells, but were almost undetectable following intraperitoneal inoculation with HCT116 and MKN45 cells (Fig. 1A). Numerous metastatic nodules were detected in the mesentery of all mice (Fig. 1B).

Antitumor activity of TFTD in the human colorectal intraperitoneal xenograft model. Fig. 2A and B presents the survival curve and body weight of nude mice intraperitoneally inoculated with DLD-1 cells, respectively. Untreated control mice succumbed to peritoneal dissemination after $\sim 7$ weeks with bloody ascites. The mice treated with 5FU, S-1, CPT-11 and TFTD exhibited a significantly longer survival time compared with untreated mice $(\mathrm{P}<0.01$; Fig. $2 \mathrm{~A}$ and Table I). No significant difference in mean body weight of mice treated with these drugs compared with that of untreated mice was 
Table I. Antitumor activity of TFTD in the human colorectal intraperitoneal xenograft model.

Survival day, median (ILS, \%)

\begin{tabular}{lccccc}
\cline { 2 - 5 } Cell line & Control & TFTD & S-1 & 5FU & CPT-11 \\
\hline DLD-1 & $51(-)$ & $85^{\mathrm{a}-\mathrm{c}}(67)$ & $68^{\mathrm{a}}(32)$ & $65^{\mathrm{a}}(26)$ & $87^{\mathrm{a}-\mathrm{c}}(70)$ \\
DLD-1/5FU & $64(-)$ & $91^{\mathrm{a}-\mathrm{c}}(43)$ & $64(0.80)$ & $65(1)$ & $109^{\mathrm{a}-\mathrm{c}, \mathrm{e}}(70)$ \\
HT-29 & $40(-)$ & $83^{\mathrm{a}-\mathrm{d}}(106)$ & $64^{\mathrm{a}, \mathrm{c}}(60)$ & $58^{\mathrm{a}}(44)$ & $69^{\mathrm{a}, \mathrm{c}}(73)$ \\
HCT116 & $29(-)$ & $58^{\mathrm{a}, \mathrm{b}}(98)$ & $34^{\mathrm{a}}(17)$ & n.d. & $54^{\mathrm{a}}(84)$ \\
\hline
\end{tabular}

ILS, increase in lifespan $(\%)=[($ median survival time of treated group $) /($ median survival time of control group $)-1] x 100$. n.d., not determined. ${ }^{\mathrm{a}} \mathrm{P}<0.01$ vs. control; ${ }^{\mathrm{P}}<0.01$ vs. $\mathrm{S}-1$ group; ${ }^{\mathrm{C}} \mathrm{P}<0.01$ vs. $5 \mathrm{FU}$ group; ${ }^{\mathrm{d}} \mathrm{P}<0.01 \mathrm{vs}$. CPT- 11 group; ${ }^{\mathrm{e}} \mathrm{P}<0.01$ vs. TFTD group. TFTD, trifluridine/tipiracil; 5FU, 5-fluorouracil; S-1, tegafur, gimeracil and potassium oxonate; CPT-11, irinotecan.
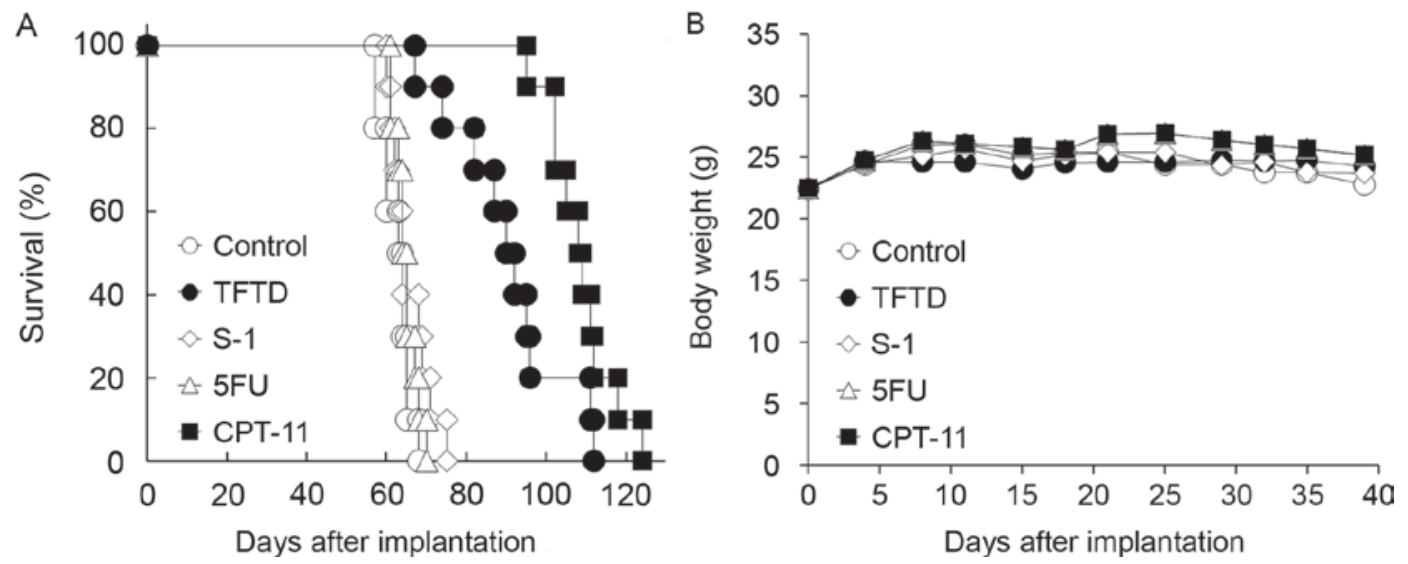

Figure 3. Effect of TFTD (A) on the survival of mice intraperitoneally transplanted with human colorectal cancer DLD-1/5FU cells and (B) on the body weight of DLD-1/5FU tumor-bearing nude mice. Mice were treated with (०) vehicle, $(\bullet)$ TFTD (200 mg/kg, orally twice daily for 5 consecutive days followed by 2 drug-free days for 6 weeks), ( () S-1 (10 mg/kg/day, orally once daily for 5 consecutive days followed by 2 drug-free days for 6 weeks), $(\Delta) 5 \mathrm{FU}(12 \mathrm{mg} / \mathrm{kg}$, intravenous injection three times a week every three weeks for 6 weeks) and (- CPT-11 (100 mg/kg, intravenously once weekly for 6 weeks). Body weight was measured twice weekly. Results are presented as means $(\mathrm{n}=10)$. TFTD, trifluridine/tipiracil; S-1, tegafur, gimeracil and potassium oxonate; 5FU, 5-fluorouracil; CPT-11, irinotecan.

identified (Fig. 2B). The results presented in Table I indicate that, in the DLD-1 model, the mice treated with TFTD or CPT-11 exhibited the most improved survival. The median ILS of mice treated with TFTD and CPT-11 was 85 days and 87 days, respectively. The mice treated with TFTD and CPT-11 exhibited significantly increased survival times compared with the mice treated with $5 \mathrm{FU}$ or $\mathrm{S}-1(\mathrm{P}<0.01$; Table I).

In the DLD-1/5FU intraperitoneal xenograft model, no significant antitumor effect of $5 \mathrm{FU}$ or S-1 was identified compared with the untreated control group (Fig. 3A and Table I). TFTD exhibited a significant antitumor activity compared with that of the untreated control group $(\mathrm{P}<0.01)$, but the effect of TFTD was weaker compared with that of CPT-11 in the DLD-1/5FU model (Table I). No significant body weight loss of drug treated groups was identified compared with those in the untreated control group (Fig. 3B).

In contrast, TFTD exhibited a significant antitumor effect when compared with CPT-11 in the HT-29 intraperitoneal xenograft model $(\mathrm{P}<0.01$; Fig. $4 \mathrm{~A}$ and Table I). TFTD also exhibited a significant antitumor effect when compared with the 5FU and S-1 groups in the HT-29 xenograft model $(\mathrm{P}<0.01$; Table I). In HT-29 cells, no significant body weight loss in the drug treated groups was identified compared with those in the untreated control group (Fig. 4B).

TFTD, S-1 and CPT-11 significantly increased the survival in the HCT116 xenograft model $(\mathrm{P}<0.01$; Fig. 5 and Table I). TFTD and CPT-11 exhibited significant antitumor activities compared with S-1 in the HCT116 xenograft model $(\mathrm{P}<0.01$; Table I). In the HCT116 intraperitoneal xenograft models, no significant body weight loss of drug treated groups were identified compared with those in the untreated control group (Fig. 5B).

Antitumor activity of TFTD in the human gastric MKN45 intraperitoneal xenograft model. Fig. 6A and B present the survival curve and body weight of nude mice inoculated with the human gastric MKN45 cells, respectively. Untreated control mice succumbed to peritoneal dissemination after $\sim 4$ weeks. TFTD exhibited a significant antitumor effect compared with S-1, 5FU and CDDP in the MKN45 intraperitoneal xenograft model ( $\mathrm{P}<0.01$; Table II). The body weight in the control group gradually decreased during the dosing period. No significant difference in weight loss of the drug-treated mice (TFTD, S-1 and CDDP) was identified compared with that of the control 

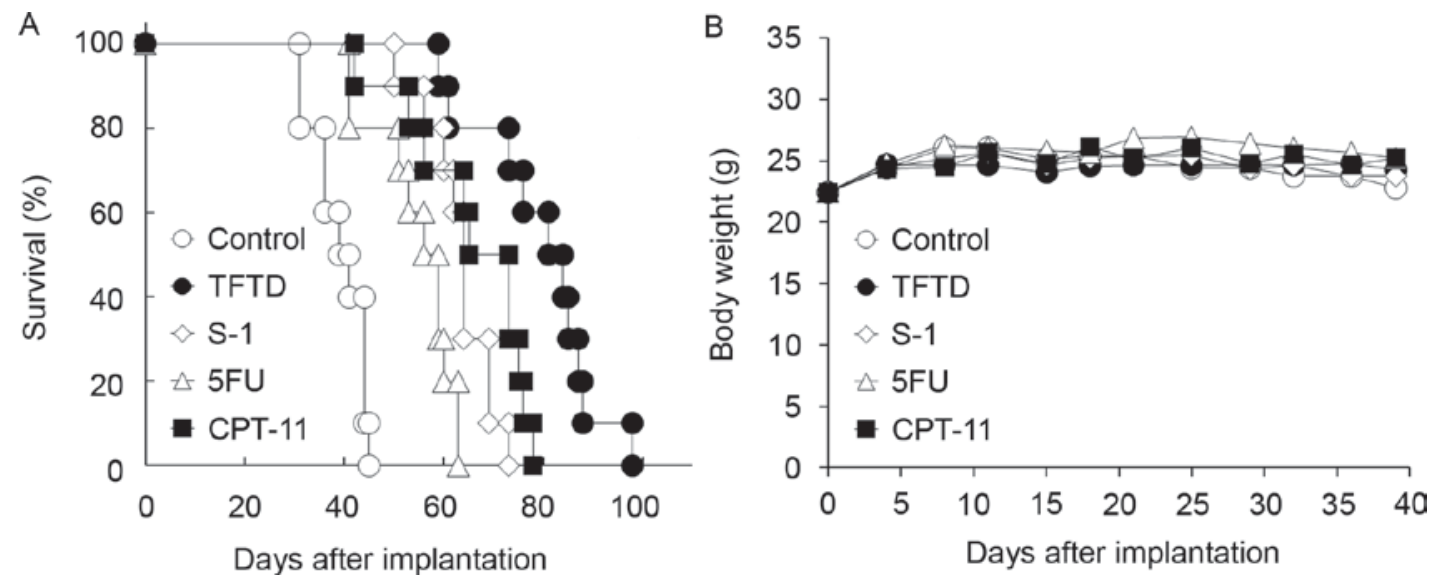

Figure 4. Effect of TFTD (A) on the survival of mice intraperitoneally transplanted with human colorectal cancer HT-29 cells and (B) on body weight in HT-29 tumor-bearing nude mice. Mice were treated with (o) vehicle, (•) TFTD $(200 \mathrm{mg} / \mathrm{kg}$, orally twice daily for 5 consecutive days followed by 2 drug-free days for 6 weeks), ( $)$ S-1 (10 mg/kg/day, orally once daily for 5 consecutive days followed by 2 drug-free days for 6 weeks), ( $\Delta$ ) $5 \mathrm{FU}$ (12 mg/kg, intravenous injection three times a week every 3 weeks for 6 weeks) and (- CPT-11 (100 mg/kg, intravenously once weekly for 6 weeks). Body weight was measured twice weekly. Results are presented as means ( $\mathrm{n}=10$ ). TFTD, trifluridine/tipiracil; 5FU, 5 -fluorouracil; S-1, tegafur, gimeracil and potassium oxonate; CPT-11, irinotecan.
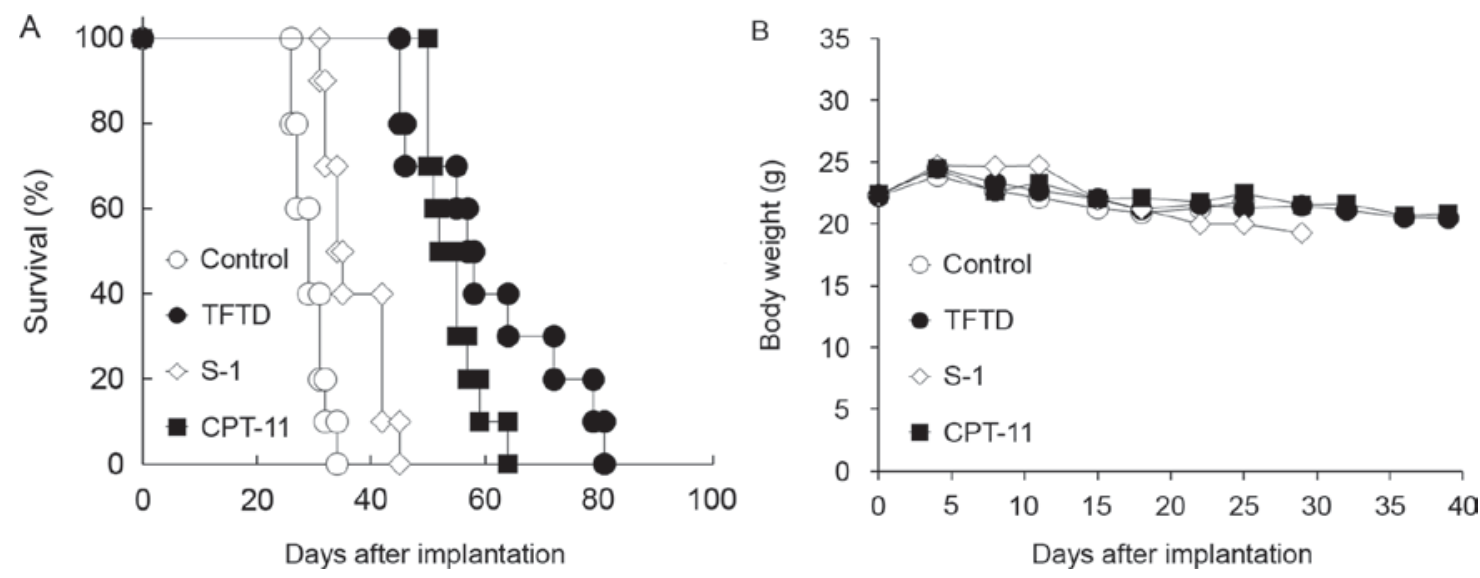

Figure 5. Effect of TFTD (A) on the survival of mice intraperitoneally transplanted with human colorectal cancer HCT116 cells and (B) on the body weight of HCT116 tumor-bearing nude mice. Mice were treated with (०) vehicle, (•) TFTD $(200 \mathrm{mg} / \mathrm{kg}$, orally twice daily for 5 consecutive days followed by 2 drug-free days for 6 weeks), ( $\diamond)$ S-1 (10 mg/kg/day, orally once daily for 5 consecutive days followed by 2 drug-free days for 6 weeks), $(\Delta) 5 \mathrm{FU}(12 \mathrm{mg} / \mathrm{kg}$, intravenous injection three times a week every 3 weeks for 6 weeks) and (๘) CPT-11 (100 mg/kg, intravenously once weekly for 6 weeks). Body weight was measured twice weekly. Results are presented as means $(\mathrm{n}=10)$. TFTD, trifluridine/tipiracil; S-1, tegafur, gimeracil and potassium oxonate; 5FU, 5-fluorouracil; CPT-11, irinotecan.
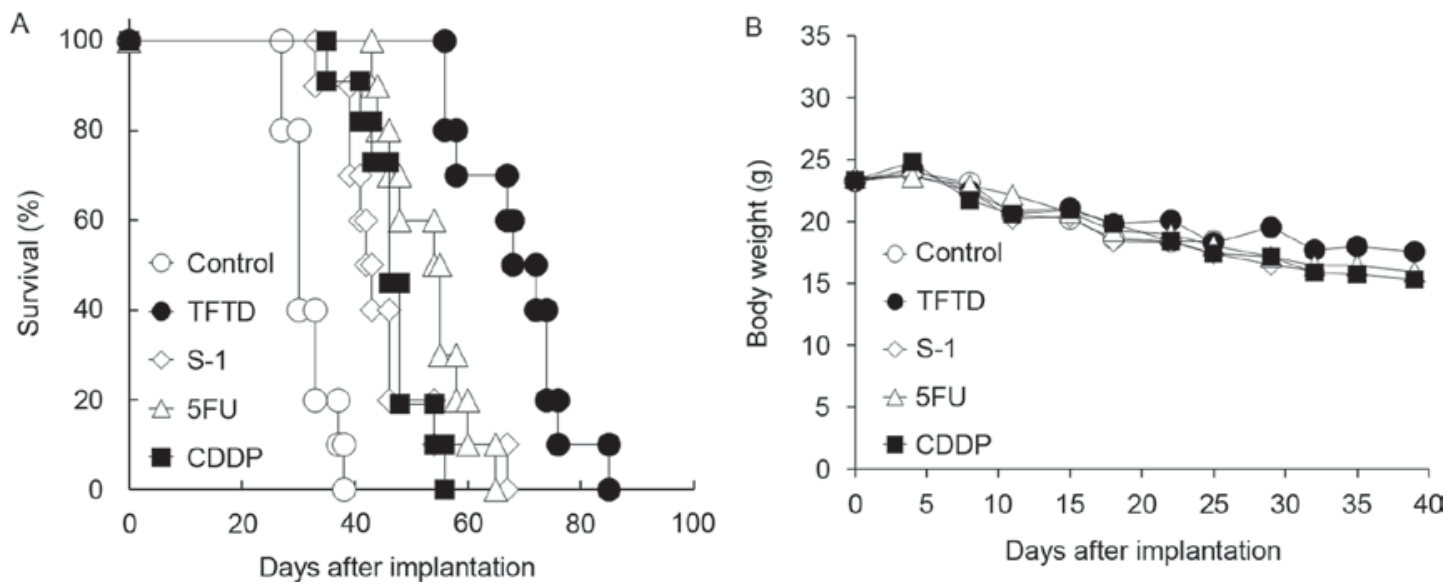

Figure 6. Effect of TFTD (A) on the survival of mice intraperitoneally transplanted with human gastric cancer MKN45 cells and (B) on the body weight of MKN45 tumor-bearing nude mice. Mice were treated with ( $($ ) vehicle, $(\bullet)$ TFTD $(200 \mathrm{mg} / \mathrm{kg}$, orally twice daily for 5 consecutive days followed by 2 drug-free days for 6 weeks), ( $\diamond)$ S-1 (10 mg/kg/day, orally once daily for 5 consecutive days followed by 2 drug-free days for 6 weeks), $(\Delta) 5 \mathrm{FU}(12 \mathrm{mg} / \mathrm{kg}$, intravenous injection three times a week every three weeks for 6 weeks) and (a) CDDP (7 mg/kg, intravenously once every three weeks for 6 weeks). Body weight was measured twice weekly. Results are presented as means $(\mathrm{n}=10)$. TFTD, trifluridine/tipiracil; S-1, tegafur, gimeracil and potassium oxonate; 5FU, 5-fluorouracil; CDDP, cisplatin. 
Table II. Antitumor activity of TFTD in the human gastric MKN45 intraperitoneal xenograft model.

Survival day, median (ILS, \%)

\begin{tabular}{lccccr}
\cline { 2 - 5 } Cell line & Control & TFTD & S-1 & 5FU & CDDP \\
\hline MKN45 & $30(-)$ & $70^{\text {acc }}(133)$ & $43^{\mathrm{a}}(42)$ & $46^{\mathrm{a}}(53)$ & $56^{\mathrm{a}}(85)$ \\
\hline
\end{tabular}

ILS, increase in lifespan $(\%)=[($ median survival time of treated group $) /($ median survival time of control group)-1]x100. n.d., not determined. ${ }^{a} \mathrm{P}<0.01$ vs. control; ${ }^{\mathrm{P}} \mathrm{P}<0.01 \mathrm{vs}$. $\mathrm{S}-1$ group; ${ }^{\mathrm{c}} \mathrm{P}<0.01$ vs. CDDP group. TFTD, trifluridine/tipiracil; $\mathrm{S}-1$, tegafur, gimeracil and potassium oxonate; 5FU, 5-fluorouracil; CDDP, cisplatin.

mice (Fig. 6B). No drug-related toxicity was detected during the present study.

Peritoneal fluid CEA levels in mice treated with TFTD and $5 F U$ in the human gastric MKN45 intraperitoneal xenograft model. On day 10 following intraperitoneal inoculation of MKN45 tumor cells, the mean CEA level in the peritoneal fluid of untreated control mice was $4,061 \mathrm{ng} / \mathrm{ml}$. The CEA level in control mice increased to $12,234 \mathrm{ng} / \mathrm{ml}$ on day 30 (Fig. 7). The CEA level in mice treated with 5FU increased from $2,855 \mathrm{ng} / \mathrm{ml}$ (70.3\% of control) on day 10 to $4,210 \mathrm{ng} / \mathrm{ml}$ (52.8\% of control) on day $20,8,665 \mathrm{ng} / \mathrm{ml}$ (70.8\% of control) on day 30 and 10,658 ng/ml on day 40 . In contrast, the CEA level in mice treated with TFTD was $1,547 \mathrm{ng} / \mathrm{ml}$ (38.1\% of control) on day $10,1,795 \mathrm{ng} / \mathrm{ml}(22.5 \%$ of control) on day 20 , and $3,284 \mathrm{ng} / \mathrm{ml}$ ( $26.8 \%$ of control) on day 30 . Almost all mice succumbed to peritoneal dissemination when the CEA level in the peritoneal fluid was $>10,000 \mathrm{ng} / \mathrm{ml}$.

\section{Discussion}

In the present study, a reliable and feasible nude mouse survival model of peritoneal dissemination of colorectal and gastric cancers was developed. Using these models, it was determined that i) TFTD treatment significantly prolonged survival in nude mice bearing intraperitoneal human colorectal tumors and human gastric tumors, and ii) the antitumor effects of TFTD were similar to those of CPT-11 and were significantly increased compared with those of 5FU, S-1 and CDDP without body weight loss.

5FU and its derivatives are uracil-based nucleic acid analogs that inhibit thymidylate synthase, which is a key enzyme in DNA synthesis, and are incorporated into nucleic acids, causing RNA damage. Unlike 5FU and its derivatives, the primary cytotoxic mechanism of trifluridine with twice-daily oral dosing is hypothesized to be DNA incorporation, causing DNA dysfunction (19). TFTD has a unique mechanism of action and is used for the treatment of patients with unresectable advanced or recurrent colorectal cancer that is refractory to standard therapies. In preclinical studies, when 5FU-resistant human tumors are transplanted subcutaneously into the dorsal region of each animal, TFTD exhibited a significant antitumor effect compared with that of 5FU and its derivatives (18). The mechanism of tumor cell resistance to $5 \mathrm{FU}$ of DLD-1/5FU is hypothesized to involve decreased incorporation of 5FU into RNA. The effect of TFTD on

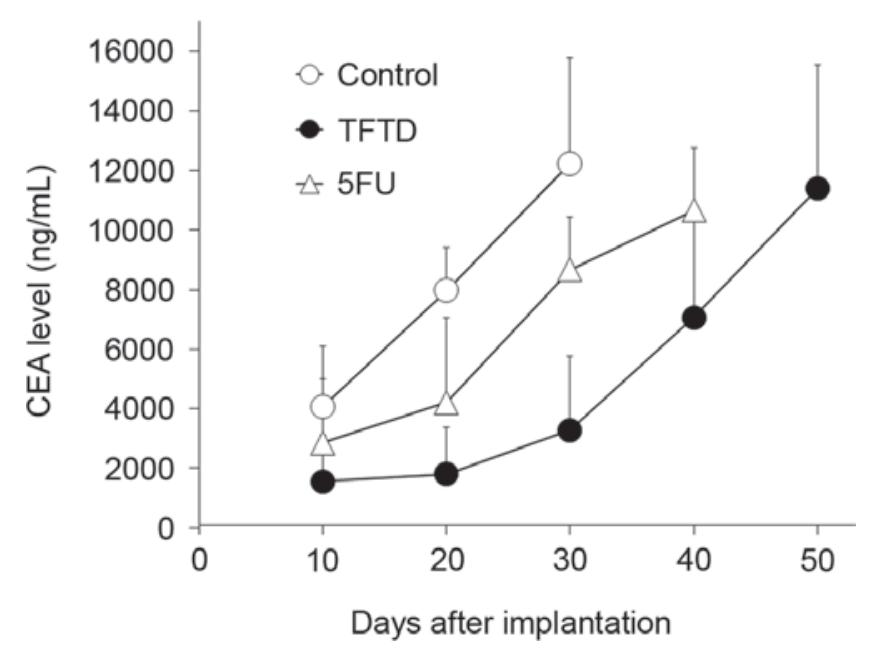

Figure 7. Mean intraperitoneal CEA level of control, TFTD- and 5FU-treated mice intraperitoneally transplanted with human gastric cancer MKN45 cells. Mice were treated with (०) vehicle, $(\bullet)$ TFTD $(200 \mathrm{mg} / \mathrm{kg}$, orally twice daily for 5 consecutive days followed by 2 drug-free days for 6 weeks) and $(\Delta) 5 F U(12 \mathrm{mg} / \mathrm{kg}$, intravenous injection three times a week every three weeks for 6 weeks). Results are presented as the means \pm standard deviation $(n=6)$. CEA, carcinoembryonic antigen; TFTD, trifluridine/tipiracil; 5FU, 5-fluorouracil.

the DLD-1/5FU intraperitoneal xenograft is consistent with previous results (18).

In the present study, four colorectal cancer cell lines and one gastric cancer cell line were used. HT-29 and MKN45 are KRAS wild-type, whereas DLD-1 and HCT116 carry a KRAS mutation. In the present study, TFTD exhibited similar anticancer activities on these colorectal or gastric cancer cell lines regardless of the KRAS status (Tables I and II). Consistent with these results, TFTD has been shown to improve overall survival in a clinical setting regardless of the KRAS status of tumors (20). Furthermore, the BRAF mutation status of DLD-1, HCT116 and MKN45 is wild-type and that of HT-29 is mutant. The p53 mutation status of DLD-1, HT-29 and MKN45 is wild-type and that of HCT116 is mutant. In the present study, TFTD also exhibited similar anticancer activities regardless of the BRAF or p53 status on these cancer cell lines. Therefore, TFTD may be beneficial to patients with mutated and wild-type KRAS tumors, and to patients with mutated and wild-type BRAF or p53 tumors.

In the present study, the effects of TFTD were identified to be significantly increased compared with those of 5FU and S-1 in nude mice bearing intraperitoneal human colorectal tumors 
and human gastric tumors. These data are consistent with results obtained using KM20C colon cancer cells (29). Trifluridine is incorporated in place of thymidine bases in DNA (30). DNA glycosylases involved in the excision of uracil and 5-FU from DNA include uracil N-glycosylase (UNG), single-strand-selective monofunctional uracil-DNA glycosylase 1 (SMUG1), thymine DNA glycosylase (TDG), and methyl-CpG-binding domain 4 (MBD4) (31). Trifluridine inserted at T-sites (paired to A) is not excised by UNG, SMUG1, TDG or MBD4 (30). The incorporated trifluridine into DNA is sustained in the DNA for a marked period, and its incorporation into DNA induces instability of the DNA (32). This persistent antitumor activity is likely to account for the ability of TFTD to prolong the survival of mice intraperitoneally transplanted with human tumor cells. The preclinical data of the present study may be useful to predict the potential clinical benefits of an anticancer agent.

The CEA levels of 5FU-treated mice between 10 and 30 days ranged between 50 and $70 \%$, whereas that of the TFTD-treated mice ranged between 20 and $40 \%$. On early days following intraperitoneal inoculation of MKN45 tumor cells, the increase in CEA levels was inhibited by TFTD. These TFTD inhibitory effects may be related to the effects of TFTD on the survival of mice transplanted with tumor cells when compared with those of other drugs. In clinical settings, TFTD may inhibit tumor relapse following surgery at an early stage of colorectal or gastric cancer in patients.

Certain anticancer agents failed to show any benefits in clinical trials in spite of being highly effective in a mouse xenograft model (33-35). The majority of clinical studies enroll advanced and late-stage patients. Conversely, almost all mouse studies do not measure therapeutic effects on advanced metastatic disease (33). The experimental model of the present study mimicked clinical studies. However, the effects of TFTD were only evaluated at a single dose $(200 \mathrm{mg} / \mathrm{kg})$ by using a single schedule (twice daily for 5 consecutive days followed by 2 drug-free days for 6 weeks) in the present study. Further investigation of the optimal dosing schedule for TFTD is required to predict the clinical response to TFTD.

Finally, in the present study, TFTD exhibited superior antitumor efficacy to that of the fluoropyrimidines and CDDP in a peritoneal dissemination mouse model using human colorectal and gastric cancer cells. The activity was also confirmed by measuring CEA levels in MKN45 tumors. A clinical randomized double-blind Phase III study evaluating TAS-102 plus BSC compared with placebo plus BSC in patients with metastatic gastric cancer refractory to standard treatments is currently ongoing (22), and its outcome is expected to be informative.

\section{References}

1. Spolverato G, Ejaz A, Azad N and Pawlik TM: Surgery for colorectal liver metastases: The evolution of determining prognosis. World J Gastrointest Oncol 5: 207-221, 2013.

2. Lemmens VE, Klaver YL, Verwaal VJ, Rutten HJ, Coebergh JW and de Hingh IH: Predictors and survival of synchronous peritoneal carcinomatosis of colorectal origin: A population-based study. Int J Cancer 128: 2717-2725, 2011.

3. Saltz LB, Clarke S, Díaz-Rubio E, Scheithauer W, Figer A, Wong R, Koski S, Lichinitser M, Yang TS, Rivera F, et al: Bevacizumab in combination with oxaliplatin-based chemotherapy as first-line therapy in metastatic colorectal cancer: A randomized phase III study. J Clin Oncol 26: 2013-2019, 2008.
4. Sobrero AF, Maurel J, Fehrenbacher L, Scheithauer W, Abubakr YA, Lutz MP, Vega-Villegas ME, Eng C, Steinhauer EU, Prausova J, et al: EPIC: Phase III trial of cetuximab plus irinotecan after fluoropyrimidine and oxaliplatin failure in patients with metastatic colorectal cancer. J Clin Oncol 26: 2311-2319, 2008.

5. Goldberg RM, Sargent DJ, Morton RF, Fuchs CS, Ramanathan RK, Williamson SK, Findlay BP, Pitot HC and Alberts S: Randomized controlled trial of reduced-dose bolus fluorouracil plus leucovorin and irinotecan or infused fluorouracil plus leucovorin and oxaliplatin in patients with previously untreated metastatic colorectal cancer: A North American Intergroup Trial. J Clin Oncol 24: 3347-3353, 2006.

6. Hurwitz H, Fehrenbacher L, Novotny W, Cartwright T, Hainsworth J, Heim W, Berlin J, Baron A, Griffing S, Holmgren E, et al: Bevacizumab plus irinotecan, fluorouracil, and leucovorin for metastatic colorectal cancer. N Engl J Med 350: 2335-2342, 2004

7. ClinicalTrials.gov ClinicalTrials.gov Identifier: NCT00003594: Combination Chemotherapy in Treating Patients With Advanced Colorectal Cancer, http://clinicaltrials.gov/show/NCT00003594. Accessed March 10, 2017

8. ClinicalTrials.gov ClinicalTrials.gov Identifier: NCT00005036: Irinotecan Compared With Combination Chemotherapy in Treating Patients With Advanced Colorectal Cancer. http://clinicaltrials.gov/show/NCT00005036, Accessed March 10, 2017.

9. Franko J, Shi Q, Goldman CD, Pockaj BA, Nelson GD, Goldberg RM, Pitot HC, Grothey A, Alberts SR and Sargent DJ: Treatment of colorectal peritoneal carcinomatosis with systemic chemotherapy: A pooled analysis of north central cancer treatment group phase III trials N9741 and N9841. J Clin Oncol 30: 263-267, 2012.

10. Maehara Y, Hasuda S, Koga T, Tokunaga E, Kakeji Y and Sugimachi K: Postoperative outcome and sites of recurrence in patients following curative resection of gastric cancer. Br J Surg 87: 353-357, 2000.

11. Sadeghi B, Arvieux C, Glehen O, Beaujard AC, Rivoire M, Baulieux J, Fontaumard E, Brachet A, Caillot JL, Faure JL, et al: Peritoneal carcinomatosis from non-gynecologic malignancies: Results of the EVOCAPE 1 multicentric prospective study. Cancer 88: 358-363, 2000.

12. Yamaguchi H, Kitayama J, Ishigami $H$, Emoto S, Yamashita $H$ and Watanabe T: A phase 2 trial of intravenous and intraperitoneal paclitaxel combined with S-1 for treatment of gastric cancer with macroscopic peritoneal metastasis. Cancer 119: 3354-3358, 2013.

13. Heidelberger C, Birnie GD, Boohar J and Wentland D: Fluorinated pyrimidines. XX. Inhibition of the nucleoside phosphorylase cleavage of 5-fluoro-2'-deoxyuridine by 5-trifluoromethyl-2'-deoxyuridine. Biochim Biophys Acta 76: 315-318, 1963.

14. Gottschling $\mathrm{H}$ and Heidelberger $\mathrm{C}$ : Fluorinated pyrimidines: XIX some biological effects of 5-trifluoromthyluracil and 5-trifluoromethyl-2'-deoxyuridine of Escherichia coli and bacteriophage T4 G. J Mol Biol 7: 541-560, 1963.

15. Reyes P and Heidelberger C: Fluorinated pyrimidines. XXVI. Mammalian thymidylate synthetase: Its mechanism of action and inhibition by fluorinated nucleotides. Mol Pharmacol 1: 14-30, 1965.

16. Fujiwara Y, Oki T and Heidelberger C: Fluorinated pyrimidines. XXXVII. Effects of 5-trifluoromethyl-2'-deoxyuridine on the synthesis of deoxyribonucleic acid of mammalian cells in culture. Mol Pharmacol 6: 273-280, 1970.

17. Fukushima M, Suzuki N, Emura T, Yano S, Kazuno H, Tada Y, Yamada Y and Asao T: Structure and activity of specific inhibitors of thymidine phosphorylase to potentiate the function of antitumor 2'-deoxyribonucleosides. Biochem Pharmacol 59: 1227-1236, 2000.

18. Emura T, Suzuki N, Yamaguchi M, Ohshimo H and Fukushima M: A novel combination antimetabolite, TAS-102, exhibits antitumor activity in FU-resistant human cancer cells through a mechanism involving FTD incorporation in DNA. Int J Oncol 25: 571-578, 2004.

19. Lenz HJ, Stintzing S and Loupakis F: TAS-102, a novel antitumor agent: A review of the mechanism of action. Cancer Treat Rev 41: 777-783, 2015.

20. Mayer RJ, Van Cutsem E, Falcone A, Yoshino T, Garcia-Carbonero R, Mizunuma N, Yamazaki K, Shimada Y, Tabernero J, Komatsu Y, et al: Randomized trial of TAS-102 for refractory metastatic colorectal cancer. N Engl J Med 372: 1909-1919, 2015. 
21. Bando H, Doi T, Muro K, Yasui H, Nishina T, Yamaguchi K, Takahashi S, Nomura S, Kuno H, Shitara K, et al: A multicenter phase II study of TAS-102 monotherapy in patients with pre-treated advanced gastric cancer (EPOC1201). Eur J Cancer 62: 46-53, 2016.

22. ClinicalTrials.gov ClinicalTrials.gov Identifier: NCT02500043: Study of TAS-102 or Placebo Plus BSC in Patients With Metastatic Gastric Cancer. http://clinicaltrials.gov/show/NCT02500043. Accessed March 10, 2017.

23. Murakami Y, Kazuno H, Emura T, Tsujimoto H, Suzuki N and Fukushima M: Different mechanisms of acquired resistance to fluorinated pyrimidines in human colorectal cancer cells. Int J Oncol 17: 277-283, 2000

24. Motoyama T, Hojo $\mathrm{H}$ and Watanabe $\mathrm{H}$ : Comparison of seven cell lines derived from human gastric carcinomas. Acta Pathol Jpn 36: 65-83, 1986.

25. Kasuya K, Nagakawa Y, Suzuki M, Suzuki Y, Kyo B, Suzuki S Matsudo T, Itoi T, Tsuchida A and Aoki T: Combination therapy of gemcitabine or oral S-1 with the anti-VEGF monoclonal antibody bevacizumab for pancreatic neuroendocrine carcinoma Exp Ther Med 3: 599-602, 2012.

26. Guo XF, Yang ZR, Wang J, Lei XF, Lv XG and Dong WG: Synergistic antitumor effect of puerarin combined with 5-fluorouracil on gastric carcinoma. Mol Med Rep 11: 2562-2568, 2015.

27. Kawato Y, Furuta T, Aonuma M, Yasuoka M, Yokokura T and Matsumoto K: Antitumor activity of a camptothecin derivative, CPT-11, against human tumor xenografts in nude mice. Cancer Chemother Pharmacol 28: 192-198, 1991.

28. Arjumand W and Sultana S: Glycyrrhizic acid: A phytochemical with a protective role against cisplatin-induced genotoxicity and nephrotoxicity. Life Sci 89: 422-429, 2011.
29. Tanaka N, Sakamoto K, Okabe H, Fujioka A, Yamamura K, Nakagawa F, Nagase H, Yokogawa T, Oguchi K, Ishida K, et al: Repeated oral dosing of TAS-102 confers high trifluridine incorporation into DNA and sustained antitumor activity in mouse models. Oncol Rep 32: 2319-2326, 2014.

30. Suzuki N, Emura T and Fukushima M: Mode of action of trifluorothymidine (TFT) against DNA replication and repair enzymes. Int J Oncol 39: 263-270, 2011.

31. Grogan BC, Parker JB, Guminski AF and Stivers JT: Effect of the thymidylate synthase inhibitors on dUTP and TTP pool levels and the activities of DNA repair glycosylases on uracil and 5-fluorouracil in DNA. Biochemistry 50: 618-627, 2011.

32. Markley JC, Chirakul P, Sologub D and Sigurdsson ST: Incorporation of 2'-deoxy-5-(trifluoromethyl)uridine and 5-cyano-2'-deoxyuridine into DNA. Bioorg Med Chem Lett 11: 2453-2455, 2001.

33. Kerbel RS: Human tumor xenografts as predictive preclinical models for anticancer drug activity in humans: Better than commonly perceived-but they can be improved. Cancer Biol Ther 2 (4 Suppl 1): S134-S139, 2003.

34. Zhang C, Awasthi N, Schwarz MA and Schwarz RE: Establishing a peritoneal dissemination xenograft mouse model for survival outcome assessment of experimental gastric cancer. J Surg Res 182: 227-234, 2013.

35. Gould SE, Junttila MR and de Sauvage FJ: Translational value of mouse models in oncology drug development. Nat Med 21: 431-439, 2015 